\title{
Hexosome and Hexagonal Phases Mediated by Hydration and Polymeric Stabilizer
}

\author{
Idit Amar-Yuli ${ }^{1}$ Ellen Wachtel ${ }^{2}$,Einav Ben Shoshan ${ }^{3}$, Dganit Danino ${ }^{3}$, \\ Abraham Aserin ${ }^{1}$, Nissim Garti ${ }^{1}$ * \\ ${ }^{1}$ Casali Institute of Applied Chemistry, The Hebrew University of Jerusalem, \\ Jerusalem 91904, Israel \\ ${ }^{2}$ Faculty of Chemistry, The Weizmann Institute of Science, Rehovot 76100, Israel \\ ${ }^{3}$ Department of Biotechnology and Food Engineering, Technion-Israel Institute of \\ Technology, Haifa 32000, Israel
}

e-mail: garti@vms.huji.ac.il 
Structural properties of the less ordered and more ordered (10 - $25 \mathrm{wt} \%$ water concentrations) hexagonal structures upon addition of an amphiphilic block copolymer (F127).

\begin{tabular}{|c|c|c|c|}
\hline \multicolumn{2}{|c|}{$\begin{array}{l}\text { Lipid/polymer weight ratio } \\
\text { (wt\% polymer) }\end{array}$} & \multirow{2}{*}{$\begin{array}{c}\begin{array}{c}\mathrm{a}(\AA) \\
\pm 0.5 \AA\end{array} \\
46.9\end{array}$} & \multirow{2}{*}{$\begin{array}{r}\begin{array}{c}\mathrm{L}_{\mathrm{H}}(\AA) \\
+50 \AA\end{array} \\
564\end{array}$} \\
\hline \multirow{4}{*}{$10 \mathrm{wt} \%$ water } & $\begin{array}{c}100 / 0 \\
(0 \mathrm{wt} \% \text { polymer })\end{array}$ & & \\
\hline & $\begin{array}{c}95 / 5 \\
(4.5 \mathrm{wt} \% \text { polymer })\end{array}$ & $45.5^{*}$ & $422 *$ \\
\hline & $\begin{array}{c}90 / 10 \\
(9 \mathrm{wt} \% \text { polymer })\end{array}$ & \multicolumn{2}{|c|}{ w/o micellar phase } \\
\hline & $\begin{array}{c}85 / 15 \\
\text { (13.5 wt } \% \text { polymer) }\end{array}$ & \multicolumn{2}{|c|}{ w/o micellar phase } \\
\hline \multirow{4}{*}{$15 \mathrm{wt} \%$ water } & $\begin{array}{c}100 / 0 \\
(0 \mathrm{wt} \% \text { polymer })\end{array}$ & 52.3 & 772 \\
\hline & $\begin{array}{c}95 / 5 \\
(4.25 \mathrm{wt} \% \text { polymer })\end{array}$ & $49.5 *$ & $481 *$ \\
\hline & $\begin{array}{c}90 / 10 \\
(8.5 \text { wt } \% \text { polymer })\end{array}$ & \multicolumn{2}{|c|}{ w/o micellar phase } \\
\hline & $\begin{array}{c}85 / 15 \\
\text { (12.75 wt \% polymer) } \\
\end{array}$ & \multicolumn{2}{|c|}{ w/o micellar phase } \\
\hline \multirow{4}{*}{$20 \mathrm{wt} \%$ water } & $\begin{array}{c}100 / 0 \\
(0 \mathrm{wt} \% \text { polymer })\end{array}$ & 55.4 & 1014 \\
\hline & $\begin{array}{c}95 / 5 \\
(4 \mathrm{wt} \% \text { polymer })\end{array}$ & 48.2 & 647 \\
\hline & $\begin{array}{c}90 / 10 \\
\text { (8 wt } \% \text { polymer) }\end{array}$ & $48.5^{*}$ & $554 *$ \\
\hline & $\begin{array}{c}85 / 15 \\
\text { (12 wt } \% \text { polymer) }\end{array}$ & \multicolumn{2}{|c|}{ w/o micellar phase } \\
\hline \multirow{4}{*}{$25 \mathrm{wt} \%$ water } & $\begin{array}{c}100 / 0 \\
(0 \mathrm{wt} \% \text { polymer })\end{array}$ & 60.3 & 980 \\
\hline & $\begin{array}{c}95 / 5 \\
(3.75 \mathrm{wt} \% \text { polymer })\end{array}$ & 52.6 & 672 \\
\hline & $\begin{array}{c}90 / 10 \\
\text { (7.5 wt\% polymer) }\end{array}$ & 51.5 & 561 \\
\hline & $\begin{array}{c}85 / 15 \\
(11.25 \mathrm{wt} \% \text { polymer) }\end{array}$ & $49.1 *$ & $523 *$ \\
\hline
\end{tabular}

*Although only one peak is observed in SAXS measurements, we assume these phases are $\mathrm{H}_{\mathrm{II}}$, due to the typical hexagonal texture observed in the polarizing light microscope. 